\title{
Discrete-Time Recurrent Neural Networks with Time-Varying Delays: Exponential Stability Analysis
}

\author{
Yurong Liu, Zidong Wang*, Alan Serrano and Xiaohui Liu
}

\begin{abstract}
This paper is concerned with the analysis problem of exponential stability for a class of discrete-time recurrent neural networks (DRNNs) with time delays. The delay is of the time-varying nature, and the activation functions are assumed to be neither differentiable nor strict monotonic. Furthermore, the description of the activation functions is more general than the recently commonly used Lipschitz conditions. Under such mild conditions, we first prove the existence of the equilibrium point. Then, by employing a Lyapnuov-Krasovskii functional, a unified linear matrix inequality (LMI) approach is developed to establish sufficient conditions for the DRNNs to be globally exponentially stable. It is shown that the delayed DRNNs are globally exponentially stable if a certain LMI is solvable, where the feasibility of such an LMI can be easily checked by using the numerically efficient Matlab LMI Toolbox. A simulation example is presented to show the usefulness of the derived LMI-based stability condition.
\end{abstract}

\section{Keywords}

Discrete recurrent neural networks; Exponential stability; Time-varying delays; Lyapunov-Krasovskii functional; Linear matrix inequality.

\section{INTRODUCTION}

The last few decades have seen successful applications of recurrent neural networks (RNNs) to a variety of information processing systems such as signal processing, pattern recognition, optimization, model identification and associative memories, where the rich dynamical behaviors of RNNs have played a key role. It has recently been revealed that signal transmission delays may cause oscillation and instability of the neural networks (see e.g. [1]). Therefore, various analysis aspects for RNNs with delays have drawn much attention, and many results have been reported in the literature. In particular, the existence of equilibrium point, global asymptotic stability, global exponential stability, and the existence of periodic solutions have been intensively investigated, see $[2,3,16,20-24]$ for some recent publications.

Note that, up to now, most recurrent neural networks have been assumed to act in a continuous-time manner. However, when it comes to the implementation of continuous-time networks for the sake of computer-based simulation, experimentation or computation, it is usual to discretize the continuous-time networks. In fact, discrete-time neural networks have already been applied in a wide range of areas, such as image processing [4], time series analysis [10], quadratic optimization problems [18], and system identification [27], etc. In an ideal case, the discrete-time analogues should be produced in a way to reflect the dynamics of their continuoustime counterparts. Specifically, the discrete-time analogue should inherit the dynamical characteristics of

This work was supported in part by the Engineering and Physical Sciences Research Council (EPSRC) of the U.K. under Grant GR/S27658/01, the Nuffield Foundation of the U.K. under Grant NAL/00630/G, the Alexander von Humboldt Foundation of Germany, the Natural Science Foundation of Jiangsu Education Committee of China (05KJB110154), the NSF of Jiangsu Province of China (BK2006064), and the National Natural Science Foundation of China (10471119).

Y. Liu is with the Department of Mathematics, Yangzhou University, Yangzhou 225002, P. R. China.

Z. Wang, A. Serrano and X. Liu are with the Department of Information Systems and Computing, Brunel University, Uxbridge, Middlesex, UB8 3PH, United Kingdom.

${ }^{*}$ Corresponding author. Email: Zidong.Wang@brunel.ac.uk, Fax: 00441896251686. 
the continuous-time networks under mild or no restriction on the discretization step-size, and also maintain functional similarity to the continuous-time system and any physical or biological reality that the continuoustime network has [14,17]. Unfortunately, as pointed out in [15], the discretization cannot preserve the dynamics of the continuous-time counterpart even for a small sampling period. Therefore, there is a crucial need to study the dynamics of discrete-time neural networks.

Recently, the stability analysis problems for discrete-time neural networks have received considerable research interests, and various stability criteria have been proposed in the literature, see e.g. [11, 19, 26, 28-30] and the references therein. In particular, in [11], the global robust stability problem has been investigated for a general class of discrete-time interval neural networks which contain time-invariant uncertain parameters with their values being unknown but bounded in given compact sets. In [19], by means of the uncovered conditions, a set of sufficient and necessary conditions have been presented for global exponential stability of a class of generic discrete-time recurrent neural networks. Some sufficient criteria have been derived in $[26,29]$ in order to ensure the asymptotic stability of the equilibrium point for a discrete-time Cohen-Grossberg neural network model. In [30], the existence of periodic solutions has been proved for a non-autonomous discrete-time neural network by using the topological degree theory.

On the other hand, due to the fact that time-delays can change the dynamical behaviors of neural networks dramatically, discrete-time neural networks with time-delays have started to gain increasing research attention. For example, in [12], some global exponential stability criteria for the equilibrium point of discrete-time recurrent neural networks with variable delay have been presented with specific performances such as decay rate and trajectory bounds. Based on the linear matrix inequality (LMI), in [13], the uniqueness and global exponential stability of the equilibrium point have been investigated for discrete-time bi-directional associative memory (BAM) neural networks with variable delays. In [25], by using coincidence degree theory as well as a priori estimates and Lyapunov functional, the existence and global stability of periodic solution have been studied for discrete delayed high-order Hopfield-type neural networks. Very recently, in [5], a class of discretetime neural networks involving variable delays have been dealt with, and sufficient conditions on existence, uniqueness and globally exponential stability of the equilibrium point have been derived by applying M-matrix theory and some analysis techniques.

It should be pointed out that, in all the papers concerning discrete-time neural networks with time-delay mentioned above, the activation functions are assumed to satisfy the Lipschitz conditions, and the derived stability criteria are mostly delay-independent which tend to be conservative. There is still room for improvement, for example, reducing the conservatism under milder constraints. It is, therefore, our main purpose of this paper to investigate the stability analysis problem of the exponential stability for a class of delayed discrete-time recurrent neural networks under more general description on the activation functions, and obtain less conservative stability criteria by using a unified linear matrix inequality (LMI) approach. It is shown that the delayed discrete-time recurrent neural networks are globally exponentially stable if a certain LMI is solvable, where the feasibility of such an LMI can be easily checked by using the numerically efficient Matlab LMI Toolbox. A simulation example is presented to show the usefulness of the derived LMI-based stability condition.

Notations: The notations are quite standard. Throughout this paper, $\mathbb{R}^{n}$ and $\mathbb{R}^{n \times m}$ denote, respectively, the $n$-dimensional Euclidean space and the set of all $n \times m$ real matrices. The superscript " $T$ " denotes matrix transposition and the notation $X \geq Y$ (respectively, $X>Y$ ) where $X$ and $Y$ are symmetric matrices, means that $X-Y$ is positive semidefinite (respectively, positive definite). For vector or matrix $z, z \succeq 0$ means that each entry of $z$ is nonnegative. $I_{n}$ is the $n \times n$ identity matrix. $|\cdot|$ is the Euclidean norm in $\mathbb{R}^{n}$. If $A$ is a matrix, denote by $\|A\|$ its operator norm, i.e., $\|A\|=\sup \{|A x|:|x|=1\}=\sqrt{\lambda_{\max }\left(A^{T} A\right)}$ where $\lambda_{\max }(\cdot)$ (respectively, $\left.\lambda_{\min }(\cdot)\right)$ means the largest (respectively, smallest) eigenvalue of $A$. Matrices, if not explicitly specified, are assumed to have compatible dimensions. Sometimes, the arguments of a function will be omitted 
in the analysis when no confusion can arise.

\section{Problem Formulation}

Consider the following $n$-neuron discrete-time recurrent neural network (DRNN) with time delays:

$$
u_{i}(k+1)=a_{i} u_{i}(k)+\sum_{j=1}^{n} b_{i j} \hat{f}_{j}\left(u_{j}(k)\right)+\sum_{j=1}^{n} d_{i j} \hat{g}_{j}\left(u_{j}(k-\tau(k))\right)+J_{i},
$$

$i=1,2, \ldots, n$; which can be equivalently written in a vector form

$$
u(k+1)=A u(k)+B \hat{F}(u(k))+D \hat{G}(u(k-\tau(k)))+J,
$$

where $u(k)=\left(u_{1}(k), u_{2}(k), \ldots, u_{n}(k)\right)^{T}$ is the neural state vector; $A=\operatorname{diag}\left(a_{1}, a_{2}, \ldots, a_{n}\right)$ with $\left|a_{i}\right|<1$ is the state feedback coefficient matrix; the $n \times n$ matrices $B=\left[b_{i j}\right]_{n \times n}$ and $D=\left[d_{i j}\right]_{n \times n}$ are the connection weight matrix and the delayed connection weight matrix, respectively. The positive integer $\tau(k)$ denotes the time-varying delay satisfying

$$
\tau_{m} \leq \tau(k) \leq \tau_{M}, \quad k \in \mathbb{N}
$$

where $\tau_{m} \geq 0$ and $\tau_{M} \geq 0$ are known integers. In $(2), \hat{F}(u(k))=\left[\hat{f}_{1}\left(u_{1}(k)\right), \hat{f}_{2}\left(u_{2}(k)\right), \ldots, \hat{f}_{n}\left(u_{n}(k)\right)\right]^{T}$ and $\hat{G}(u(k))=\left[\hat{g}_{1}\left(u_{1}(k)\right), \hat{g}_{2}\left(u_{2}(k)\right), \ldots, \hat{g}_{n}\left(u_{n}(k)\right)\right]^{T}$ denote the neuron activation functions, and the constant vector $J=\left[J_{1}, J_{2}, \ldots, J_{n}\right]^{T}$ is the exogenous input.

It is usually assumed that the activation functions are continuous, differentiable, monotonically increasing and bounded, such as the sigmoid-type of function. However, in many electronic circuits, the input-output functions of amplifiers may be neither monotonically increasing nor continuously differentiable, hence nonmonotonic functions can be more appropriate to describe the neuron activation in designing and implementing an artificial neural network. In this paper, we make following assumptions for the neuron activation functions.

Assumption 1: For $i \in\{1,2, \ldots, n\}$, the neuron activation functions $\hat{f}_{i}(\cdot)$ and $\hat{g}_{i}(\cdot)$ in $(1)$ or $(2)$ are continuous and bounded.

Assumption 2: For $i \in\{1,2, \ldots, n\}$, the neuron activation functions in (1) or (2) satisfies

$$
\begin{aligned}
& l_{i}^{-} \leq \frac{\hat{f}_{i}\left(s_{1}\right)-\hat{f}_{i}\left(s_{2}\right)}{s_{1}-s_{2}} \leq l_{i}^{+}, \quad \forall s_{1}, s_{2} \in \mathbb{R}, \\
& v_{i}^{-} \leq \frac{\hat{g}_{i}\left(s_{1}\right)-\hat{g}_{i}\left(s_{2}\right)}{s_{1}-s_{2}} \leq v_{i}^{+}, \quad \forall s_{1}, s_{2} \in \mathbb{R},
\end{aligned}
$$

where $l_{i}^{-}, l_{i}^{+}, v_{i}^{-}, v_{i}^{+}$are some constants.

Remark 1: The constants $l_{i}^{-}, l_{i}^{+}, v_{i}^{-}, v_{i}^{+}$in Assumption 2 are allowed to be positive, negative, or zero. Hence, the resulting activation functions could be non-monotonic, and are more general than the usual sigmoid functions and the recently commonly used Lipschitz conditions. Note that with such a milder assumption, the analysis methods developed in $[5,12,13,25]$ cannot be applied directly, and a new approach will have to be developed.

Remark 2: The discrete-time recurrent neural network (1) is a discrete analog of the well-known continuoustime recurrent neural network of the form:

$$
\dot{x}_{i}(t)=-a_{i} x_{i}(t)+\sum_{j=1}^{n} b_{i j} f_{j}\left(x_{j}(t)\right)+\sum_{j=1}^{n} d_{i j} g_{j}\left(x_{j}(t-\tau(t))\right)+J_{i}, \quad i=1,2, \ldots, n,
$$

which has been investigated intensively in recent years, see $[2,3,16,20-24]$ and the references therein.

According to Assumption 1, we have the following result: 
Proposition 1: Under Assumption 1, there exists an equilibrium point for the DRNN (2).

Proof: Since the activation functions are bounded, there exists a constant $M_{0}>0$ such that $|\hat{F}(u)| \leq$ $M_{0}$ and $|\hat{G}(u)| \leq M_{0}, \forall u \in \mathbb{R}^{n}$. It follows from $\left|a_{i}\right|<1$ that $I-A$ is invertible. Now, denote $\mathcal{B}(r)=$ $\left\{u \in \mathbb{R}^{n}|| u \mid \leq r\right\}$ with $r=\left\|(I-A)^{-1}\right\|\left(\|B\| M_{0}+\|D\| M_{0}+|J|\right)$, and define the map $T: \mathcal{B} \rightarrow \mathbb{R}^{n}$ by $T(u)=(I-A)^{-1}(B \hat{F}(u)+D \hat{G}(u)+J)$. Obviously, $T$ is continuous map, and it follows readily that

$$
\begin{aligned}
|T(u)| & \leq\left\|(I-A)^{-1}\right\|(\|B\||\hat{F}(u)|+\|D\||\hat{G}(u)|+|J|) \\
& \leq\left\|(I-A)^{-1}\right\|\left(\|B\| M_{0}+\|D\| M_{0}+|J|\right) .
\end{aligned}
$$

Hence, $T$ maps $\mathcal{B}(r)$ into itself, i.e., $T: \mathcal{B}(r) \rightarrow \mathcal{B}(r)$. By Brower' Fixed Point theorem, one can infer that there exists a fixed point $u^{*}$ of $T$. Namely,

$$
(I-A)^{-1}\left(B \hat{F}\left(u^{*}\right)+D \hat{G}\left(u^{*}\right)+J\right)=u^{*},
$$

or equivalently,

$$
u^{*}=A u^{*}+B \hat{F}\left(u^{*}\right)+D \hat{G}\left(u^{*}\right)+J .
$$

Therefore, there exists an equilibrium point of DRNN (2).

Obviously, if we could prove the global stability of the neural network, then the equilibrium point is unique as well. For this purpose, we need the following definition.

Definition 1: Let $u^{*}=\left[u_{1}^{*}, u_{2}^{*}, \ldots, u_{n}^{*}\right]^{T}$ be a equilibrium point of the DRNN (2). Then, the DRNN (2) is said to be globally exponentially stable if there exist constants $\mu>0$ and $0<\alpha<1$ such that every solution of the DRNN (2) satisfies

$$
\left|u(k)-u^{*}\right| \leq \mu \alpha^{k} \max _{-\tau_{M} \leq j \leq 0}\left|u(j)-u^{*}\right|, \quad k \geq 0 .
$$

In the rest of this paper, we will focus on the stability analysis of the DRNN (2). By utilizing LyapunovKrasoviskii functional, we aim to develop an LMI approach for deriving easy-to-test sufficient conditions under which the DRNN (2) is globally exponentially stable.

\section{MAIN RESUlts}

In this section, we shall establish our stability criteria based on the LMI approach.

For presentation convenience, in the following, we denote

$$
\begin{aligned}
& L_{1}=\operatorname{diag}\left(l_{1}^{+} l_{1}^{-1}, l_{2}^{+} l_{2}^{-1}, \ldots, l_{n}^{+} l_{n}^{-1}\right), \quad L_{2}=\operatorname{diag}\left(-\frac{l_{1}^{+}+l_{1}^{-1}}{2},-\frac{l_{2}^{+}+l_{2}^{-1}}{2}, \ldots,-\frac{l_{n}^{+}+l_{n}^{-1}}{2}\right), \\
& \Upsilon_{1}=\operatorname{diag}\left(v_{1}^{+} v_{1}^{-1}, v_{2}^{+} v_{2}^{-1}, \ldots, v_{n}^{+} v_{n}^{-1}\right), \quad \Upsilon_{2}=\operatorname{diag}\left(-\frac{v_{1}^{+}+v_{1}^{-1}}{2},-\frac{v_{2}^{+}+v_{2}^{-1}}{2}, \ldots,-\frac{v_{n}^{+}+v_{n}^{-1}}{2}\right) .
\end{aligned}
$$

Our main results are given in the following theorem.

Theorem 1: Under Assumptions 1 and 2, the DRNN (2) is globally exponentially stable if there exist two diagonal matrices $\Lambda=\operatorname{diag}\left(\lambda_{1}, \lambda_{2}, \ldots, \lambda_{n}\right)>0, \Gamma=\operatorname{diag}\left(\gamma_{1}, \gamma_{2}, \ldots, \gamma_{n}\right)>0$, and two positive definite matrices $P$ and $Q$ such that the following LMI holds:

$$
\Phi=\left[\begin{array}{cccc}
\Pi & 0 & A^{T} P B-\Lambda L_{2} & A^{T} P D \\
0 & -Q-\Gamma \Upsilon_{1} & 0 & -\Gamma \Upsilon_{2} \\
B^{T} P A-\Lambda L_{2} & 0 & B^{T} P B-\Lambda & B^{T} P D \\
D^{T} P A & -\Gamma \Upsilon_{2} & D^{T} P B & D^{T} P D-\Gamma
\end{array}\right]<0
$$

with

$$
\Pi=A^{T} P A-P+\left(\tau_{M}-\tau_{m}+1\right) Q-\Lambda L_{1}
$$


Proof: First, by Proposition 1, the DRNN (2) has an equilibrium point $u^{*}$. For convenience, we shift the equilibrium $u^{*}$ to the origin by letting $x(k)=u(k)-u^{*}$, and then the DRNN (2) can be transformed into

$$
x(k+1)=A x(k)+B F(x(k))+D G(x(k-\tau(k))),
$$

where $x(k)=\left[x_{1}(k), x_{2}(k), \ldots, x_{n}(k)\right]^{T} \in \mathbb{R}^{n}$ is the state vector of the transformed system, and the transformed neuron activation functions are

$$
F(x(k)):=\left(f_{1}\left(x_{1}(k)\right), f_{2}\left(x_{2}(k)\right), \ldots, f_{n}\left(x_{n}(k)\right)\right)^{T}=\hat{F}(u(k))-\hat{F}\left(u^{*}\right)
$$

By Assumption 2, it can be verified readily that the transformed neuron activation functions satisfy

$$
\begin{aligned}
& l_{i}^{-} \leq \frac{f_{i}\left(s_{1}\right)-f_{i}\left(s_{2}\right)}{s_{1}-s_{2}} \leq l_{i}^{+}, \\
& v_{i}^{-} \leq \frac{g_{i}\left(s_{1}\right)-g_{i}\left(s_{2}\right)}{s_{1}-s_{2}} \leq v_{i}^{+},
\end{aligned}
$$

In order to show the stability of the DRNN (2), we just need to deal with the stability of the DRNN (10).

To this end, we introduce the following Lyapunov-Krasovskii functional:

$$
V(k)=V_{1}(k)+V_{2}(k)+V_{3}(k),
$$

where

$$
\begin{aligned}
V_{1}(k) & =x^{T}(k) P x(k), \\
V_{2}(k) & =\sum_{i=k-\tau(k)}^{k-1} x^{T}(i) Q x(i), \\
V_{3}(k) & =\sum_{j=k-\tau_{M}+1}^{k-\tau_{m}} \sum_{i=j}^{k-1} x^{T}(i) Q x(i) .
\end{aligned}
$$

Calculating the difference of $V(k)$ along the DRNN (10), we have

$$
\Delta V(k)=\Delta V_{1}(k)+\Delta V_{2}(k)+\Delta V_{3}(k),
$$

where

$$
\begin{aligned}
\Delta V_{1}(k)= & V_{1}(k+1)-V_{1}(k) \\
= & {[A x(k)+B F(x(k))+D G(x(k-\tau(k)))]^{T} P } \\
& \times[A x(k)+B F(x(k))+D G(x(k-\tau(k)))]-x^{T}(k) P x(k) \\
= & x^{T}(k) A^{T} P A x(k)+F^{T}(x(k)) B^{T} P B F(x(k)) \\
& \left.\left.+G^{T}(x(k-\tau(k)))\right) D^{T} P D G(x(k-\tau(k)))\right) \\
& +2 x^{T}(k) A^{T} P B F(x(k))+2 x^{T}(k) A^{T} P D G(x(k-\tau(k))) \\
& +2 F^{T}(x(k)) B^{T} P D G(x(k-\tau(k)))-x^{T}(k) P x(k),
\end{aligned}
$$




$$
\begin{aligned}
\Delta V_{2}(k)= & V_{2}(k+1)-V_{2}(k) \\
= & \sum_{i=k+1-\tau(k+1)}^{k} x^{T}(i) Q x(i)-\sum_{i=k-\tau(k)}^{k-1} x^{T}(i) Q x(i) \\
= & x^{T}(k) Q x(k)-x^{T}(k-\tau(k)) Q x(k-\tau(k))+\sum_{i=k-\tau(k+1)+1}^{k-1} x^{T}(i) Q x(i)-\sum_{i=k-\tau(k)+1}^{k-1} x^{T}(i) Q x(i) \\
= & x^{T}(k) Q x(k)-x^{T}(k-\tau(k)) Q x(k-\tau(k))+\sum_{i=k-\tau_{m}+1}^{k-1} x^{T}(i) Q x(i) \\
& +\sum_{i=k-\tau(k+1)+1}^{k-\tau_{m}} x^{T}(i) Q x(i)-\sum_{i=k-\tau(k)+1}^{k-1} x^{T}(i) Q x(i) \\
\leq & x^{T}(k) Q x(k)-x^{T}(k-\tau(k)) Q x(k-\tau(k))+\sum_{i=k-\tau_{M}+1}^{k-\tau_{m}} x^{T}(i) Q x(i),
\end{aligned}
$$

and

$$
\begin{aligned}
\Delta V_{3}(k) & =V_{3}(k+1)-V_{3}(k) \\
& =\sum_{j=k-\tau_{M}+2}^{k-\tau_{m}+1} \sum_{i=j}^{k} x^{T}(i) Q x(i)-\sum_{j=k-\tau_{M}+1}^{k-\tau_{m}} \sum_{i=j}^{k-1} x^{T}(i) Q x(i) \\
& =\sum_{j=k-\tau_{M}+1}^{k-\tau_{m}} \sum_{i=j+1}^{k} x^{T}(i) Q x(i)-\sum_{j=k-\tau_{M}+1}^{k-\tau_{m}} \sum_{i=j}^{k-1} x^{T}(i) Q x(i) \\
& =\sum_{j=k-\tau_{M}+1}^{k-\tau_{m}}\left(x^{T}(k) Q x(k)-x^{T}(j) Q x(j)\right) \\
& =\left(\tau_{M}-\tau_{m}\right) x^{T}(k) Q x(k)-\sum_{i=k-\tau_{M}+1}^{k-\tau_{m}} x^{T}(i) Q x(i) .
\end{aligned}
$$

Substituting (18)-(21) into (17) results in

$$
\begin{aligned}
\Delta V(k)= & \left.\left.x^{T}(k) A^{T} P A x(k)+F^{T}(x(k)) B^{T} P B F(x(k))+G^{T}(x(k-\tau(k)))\right) D^{T} P D G(x(k-\tau(k)))\right) \\
& +2 x^{T}(k) A^{T} \operatorname{PBF}(x(k))+2 x^{T}(k) A^{T} P D G(x(k-\tau(k)))+2 F^{T}(x(k)) B^{T} P D G(x(k-\tau(k))) \\
& -x^{T}(k) P x(k)+\left(d_{M}-d_{m}+1\right) x^{T}(k) Q x(k)-x^{T}(k-\tau(k)) Q x(k-\tau(k)) \\
= & \xi^{T}(k) \Phi_{1} \xi(k),
\end{aligned}
$$

where

$$
\begin{aligned}
& \xi(k)=\left[\begin{array}{llll}
x^{T}(k) & x^{T}(k-d(k)) & F^{T}(x(k)) & G^{T}(x(k-\tau(k)))
\end{array}\right]^{T}, \\
& \Phi_{1}=\left[\begin{array}{cccc}
\Pi_{1} & 0 & A^{T} P B & A^{T} P D \\
0 & -Q & 0 & 0 \\
B^{T} P A & 0 & B^{T} P B & B^{T} P D \\
D^{T} P A & 0 & D^{T} P B & D^{T} P D
\end{array}\right]
\end{aligned}
$$

with $\Pi_{1}=A^{T} P A-P+\left(d_{M}-d_{m}+1\right) Q$.

From (11)-(12), we have

$$
\begin{array}{r}
\left(f_{i}\left(x_{i}(k)\right)-l_{i}^{+} x_{i}(k)\right)\left(f_{i}\left(x_{i}(k)\right)-l_{i}^{-} x_{i}(k)\right) \leq 0, \quad i=1,2, \ldots, n, \\
\left(g_{i}\left(x_{i}(k-\tau(k))\right)-v_{i}^{+} x_{i}(k-\tau(k))\right)\left(g_{i}\left(x_{i}(k-\tau(k))\right)-v_{i}^{-} x_{i}(x-\tau(k))\right) \leq 0, \quad i=1,2, \ldots, n,
\end{array}
$$


which are equivalent to

$$
\begin{aligned}
& {\left[\begin{array}{c}
x(k) \\
F(x(k))
\end{array}\right]^{T}\left[\begin{array}{cc}
l_{i}^{+} l_{i}^{-} e_{i} e_{i}^{T} & -\frac{l_{i}^{+}+l_{i}^{-}}{2} e_{i} e_{i}^{T} \\
-\frac{l_{i}^{+}+l_{i}^{-}}{2} e_{i} e_{i}^{T} & e_{i} e_{i}^{T}
\end{array}\right]\left[\begin{array}{c}
x(k) \\
F(x(k))
\end{array}\right] \leq 0, \quad k=1,2, \ldots, n,} \\
& {\left[\begin{array}{c}
x(k-\tau(k)) \\
G(x(k-\tau(k)))
\end{array}\right]^{T}\left[\begin{array}{cc}
v_{i}^{+} v_{i}^{-} e_{i} e_{i}^{T} & -\frac{v_{i}^{+}+v_{i}^{-}}{2} e_{i} e_{i}^{T} \\
-\frac{v_{i}^{+}+v_{i}^{-}}{2} e_{i} e_{i}^{T} & e_{i} e_{i}^{T}
\end{array}\right]\left[\begin{array}{c}
x(k-\tau(k)) \\
G(x(k-\tau(k)))
\end{array}\right] \leq 0, \quad k=1,2, \ldots, n,}
\end{aligned}
$$

where $e_{k}$ denotes the unit column vector having " 1 " element on its $k$ th row and zeros elsewhere.

Then, from (22), (25)-(26), one has

$$
\begin{aligned}
\Delta V(k) \leq & \xi^{T}(k) \Phi_{1} \xi(k)-\sum_{i=1}^{n} \lambda_{i}\left[\begin{array}{c}
x(k) \\
F(x(k))
\end{array}\right]^{T}\left[\begin{array}{cc}
l_{i}^{+} l_{i}^{-} e_{i} e_{i}^{T} & -\frac{l_{i}^{+}+l_{i}^{-}}{2} e_{i} e_{i}^{T} \\
-\frac{l_{i}^{+}+l_{i}^{-}}{2} e_{i} e_{i}^{T} & e_{i} e_{i}^{T}
\end{array}\right]\left[\begin{array}{c}
x(k) \\
F(x(k))
\end{array}\right] \\
& -\sum_{i=1}^{n} \gamma_{i}\left[\begin{array}{c}
x(k-\tau(k)) \\
G(x(k-\tau(k)))
\end{array}\right]^{T}\left[\begin{array}{cc}
v_{i}^{+} v_{i}^{-} e_{i} e_{i}^{T} & -\frac{v_{i}^{+}+v_{i}^{-}}{2} e_{i} e_{i}^{T} \\
-\frac{v_{i}^{+}+v_{i}^{-}}{2} e_{i} e_{i}^{T} & e_{i} e_{i}^{T}
\end{array}\right]\left[\begin{array}{c}
x(k-\tau(k)) \\
G(x(k-\tau(k)))
\end{array}\right] \\
= & \xi^{T}(k) \Phi_{1} \xi(k)-\left[\begin{array}{c}
x(k) \\
F(x(k))
\end{array}\right]^{T}\left[\begin{array}{cc}
\Lambda L_{1} & \Lambda L_{2} \\
\Lambda L_{2} & \Lambda
\end{array}\right]\left[\begin{array}{c}
x(k) \\
F(x(k))
\end{array}\right] \\
& -\left[\begin{array}{c}
x(k-\tau(k)) \\
G(x(k-\tau(k)))
\end{array}\right]^{T}\left[\begin{array}{cc}
\Gamma \Upsilon_{1} & \Gamma \Upsilon_{2} \\
\Gamma \Upsilon_{2} & \Gamma
\end{array}\right]\left[\begin{array}{c}
x(k-\tau(k)) \\
G(x(k-\tau(k)))
\end{array}\right] \\
= & \xi^{T}(k) \Phi \xi(k) .
\end{aligned}
$$

Let $\lambda^{*}=\lambda_{\max }(\Phi)$. Then, from the inequality (9) and (27), it is clear that $\lambda^{*}<0$, and

$$
\Delta V(k) \leq \lambda^{*}|x(k)|^{2} .
$$

Now, we are in a position to establish the exponential stability of the DRNN (10).

First, by the definition of $V(k)$, it is readily verified that

$$
V(k) \leq \rho_{1}|x(k)|^{2}+\rho_{2} \sum_{i=k-\tau_{M}}^{k-1}|x(i)|^{2},
$$

where

$$
\rho_{1}=\lambda_{\max }(P), \quad \rho_{2}=\left(\tau_{M}-\tau_{m}+1\right) \lambda_{\max }(Q) .
$$

For any scalar $\mu>1$, the inequality (29), together with (28), implies that

$$
\begin{aligned}
& \mu^{k+1} V(k+1)-\mu^{k} V(k) \\
= & \mu^{k+1} \Delta V(k)+\mu^{k}(\mu-1) V(k) \\
\leq & \omega_{1}(\mu) \mu^{k}|x(k)|^{2}+\omega_{2}(\mu) \sum_{i=k-d_{M}}^{k-1} \mu^{k}|x(i)|^{2},
\end{aligned}
$$

where

$$
\omega_{1}(\mu)=\mu \lambda^{*}+(\mu-1) \rho_{1}, \omega_{2}(\mu)=(\mu-1) \rho_{2} .
$$

Furthermore, for any integer $N \geq \tau_{M}+1$, summing up both sides of (31) from 0 to $N-1$ with respect to $k$, we have

$$
\mu^{N} V(N)-V(0) \leq \omega_{1}(\mu) \sum_{k=0}^{N-1} \mu^{k}|x(k)|^{2}+\omega_{2}(\mu) \sum_{k=0}^{N-1} \sum_{i=k-d_{M}}^{k-1} \mu^{k}|x(i)|^{2}
$$


Note that for $\tau_{M} \geq 1$,

$$
\begin{aligned}
\sum_{k=0}^{N-1} \sum_{i=k-\tau_{M}}^{k-1} \mu^{k}|x(i)|^{2} & \leq\left(\sum_{i=-\tau_{M}}^{-1} \sum_{k=0}^{i+\tau_{M}}+\sum_{i=0}^{N-1-\tau_{M}} \sum_{k=i+1}^{i+\tau_{M}}+\sum_{i=N-\tau_{M}}^{N-1} \sum_{k=i+1}^{N-1}\right) \mu^{k}|x(i)|^{2} \\
& \leq \tau_{M} \sum_{i=-\tau_{M}}^{-1} \mu^{i+\tau_{M}}|x(i)|^{2}+\tau_{M} \sum_{i=0}^{N-1-\tau_{M}} \mu^{i+\tau_{M}}|x(i)|^{2}+\tau_{M} \sum_{i=N-1-\tau_{M}}^{N-1} \mu^{i+\tau_{M}}|x(i)|^{2} \\
& \leq \tau_{M} \mu^{\tau_{M}} \max _{-\tau_{M} \leq i \leq 0}|x(i)|^{2}+\tau_{M} \mu^{\tau_{M}} \sum_{i=0}^{N-1} \mu^{i}|x(i)|^{2}
\end{aligned}
$$

Then, from (32) and (33), one has

$$
\mu^{N} V(N) \leq V(0)+\left[\omega_{1}(\mu)+d_{M} \mu^{d_{M}} \omega_{2}(\mu)\right] \sum_{k=0}^{N-1} \mu^{k}|x(k)|^{2}+\tau_{M} \mu^{\tau_{M}} \omega_{2}(\mu) \max _{-\tau_{M} \leq i \leq 0}|x(i)|^{2} .
$$

Let $\rho_{0}=\lambda_{\min }(P)$, and $\rho=\max \left\{\rho_{1}, \rho_{2}\right\}$. It is obvious that

$$
V(N) \geq \rho_{0}|x(N)|^{2}
$$

It also follows easily from (29) that

$$
V(0) \leq \rho \max _{-d_{M} \leq i \leq 0}|x(i)|^{2} .
$$

In addition, it can be verified that there exists a scalar $\mu_{0}>1$ such that

$$
\omega_{1}\left(\mu_{0}\right)+\tau_{M} \mu_{0}^{\tau_{M}} \omega_{2}\left(\mu_{0}\right)=0
$$

Substituting (35)-(37) into (34), we obtain

$$
|x(N)|^{2} \leq \frac{1}{\rho_{0}}\left(\rho+\tau_{M} \mu_{0}^{\tau_{M}} \omega_{2}\left(\mu_{0}\right)\right)\left(\frac{1}{\mu_{0}}\right)^{N} \max _{-d_{M} \leq i \leq 0}|x(i)|^{2},
$$

which indicates that the DRNN (10) is exponentially stable. This completes the proof of the theorem.

The following corollary is an easy consequence of Theorem 1.

Corollary 1: Suppose that $\tau(k) \equiv \tau_{0}$ where $\tau_{0}>0$ is a constant scalar. Then, under Assumptions 1 and 2, the DRNN (2) is globally exponentially stable if there exist two diagonal matrices $\Lambda=\operatorname{diag}\left(\lambda_{1}, \lambda_{2}, \ldots, \lambda_{n}\right)>0$ and $\Gamma=\operatorname{diag}\left(\gamma_{1}, \gamma_{2}, \ldots, \gamma_{n}\right)>0$, and two positive definite matrices $P$ and $Q$ such that the following LMI holds:

$$
\hat{\Phi}=\left[\begin{array}{cccc}
\hat{\Pi} & 0 & A^{T} P B-\Lambda L_{2} & A^{T} P D \\
0 & -Q-\Gamma \Upsilon_{1} & 0 & -\Gamma \Upsilon_{2} \\
B^{T} P A-\Lambda L_{2} & 0 & B^{T} P B-\Lambda & B^{T} P D \\
D^{T} P A & -\Gamma \Upsilon_{2} & D^{T} P B & D^{T} P D-\Gamma
\end{array}\right]<0
$$

with

$$
\hat{\Pi}=A^{T} P A-P+Q-\Lambda L_{1} .
$$

Remark 3: In our main results, the stability analysis problems are dealt with for a general class of discretetime neural networks with time-varying delays. An LMI-based sufficient condition is derived for the stability of the neural networks addressed. The exponential stability can be readily checked by the solvability of a set of LMIs, which can be done by resorting to the Matlab LMI toolbox. Note that the LMI (9) is delay-dependent, 
hence less conservative than the traditional delay-independent conditions. In the next section, an illustrative example will be provided to show the potential of the proposed criteria.

Remark 4: It is noticed that the main results of Theorem 1 are actually dependent of the delay interval. It is known from [6-8] that such conditions might be conservative in some cases. One interesting topic for future research would be how to incorporate the idea embedded in [6-8] to obtain an improved delay-dependent result for the problem considered in this paper.

Remark 5: We would like to point out that it is possible to generalize our main results to more complex neural networks, such as neural networks with parameter uncertainties (norm-bounded uncertainties [22] and polytopic uncertainties [9]), stochastic perturbations [21-23], and Markovian jumping parameters [24]. The corresponding results will appear in the near future.

\section{Numerical Example}

In this section, a numerical example is presented to demonstrate the usefulness of the developed method on the exponential stability of the DRNN (2) with time-varying delays.

Consider the DRNN (2) with the following parameters:

$$
\begin{aligned}
& A=\left[\begin{array}{ccc}
0.4 & 0 & 0 \\
0 & 0.3 & 0 \\
0 & 0 & 0.3
\end{array}\right], B=\left[\begin{array}{ccc}
0.2 & -0.2 & 0.1 \\
0 & -.3 & 0.2 \\
-0.2 & -0.1 & -0.2
\end{array}\right], D=\left[\begin{array}{ccc}
-0.2 & 0.1 & 0 \\
-0.2 & 0.3 & 0.1 \\
0.1 & -0.2 & 0.3
\end{array}\right], \\
& J=\left[\begin{array}{lll}
-0.3 & 0.2 & -0.1
\end{array}\right]^{T}, \tau(k)=4+\sin (k \pi / 2) .
\end{aligned}
$$

Take the activation functions as follows:

$$
\begin{aligned}
& \hat{f}_{1}(s)=\tanh (0.6 s), \quad \hat{f}_{2}(s)=\tanh (-0.4 s), \quad \hat{f}_{3}(s)=\tanh (-0.2 s), \\
& \hat{g}_{1}(s)=\tanh (-0.4 s), \quad \hat{g}_{2}(s)=\tanh (0.2 s), \quad \hat{g}_{3}(s)=\tanh (0.4 s) \text {. }
\end{aligned}
$$

Form the above parameters, it can be verified that $\tau_{m}=3, \tau_{M}=5$, and

$$
L_{1}=\Upsilon_{1}=\left[\begin{array}{lll}
0 & 0 & 0 \\
0 & 0 & 0 \\
0 & 0 & 0
\end{array}\right], \quad L_{2}=\left[\begin{array}{ccc}
-0.3 & 0 & 0 \\
0 & 0.2 & 0 \\
0 & 0 & 0.1
\end{array}\right], \quad \Upsilon_{2}=\left[\begin{array}{ccc}
0.2 & 0 & 0 \\
0 & -0.1 & 0 \\
0 & 0 & -0.2
\end{array}\right]
$$

By using the Matlab LMI Toolbox, we solve LMI (9) and obtain the feasible solutions as follows:

$$
\begin{aligned}
& P=\left[\begin{array}{ccc}
1.7068 & -0.1435 & 0.1041 \\
-0.1435 & 1.6765 & 0.0583 \\
0.1041 & 0.0583 & 1.7458
\end{array}\right], Q=\left[\begin{array}{ccc}
0.2711 & -0.0344 & 0.0250 \\
-0.0344 & 0.2962 & 0.0145 \\
0.0250 & 0.0145 & 0.3134
\end{array}\right] \text {, } \\
& \Lambda=\left[\begin{array}{ccc}
1.2343 & 0 & 0 \\
0 & 1.3497 & 0 \\
0 & 0 & 1.3652
\end{array}\right], \Gamma=\left[\begin{array}{ccc}
1.3331 & 0 & 0 \\
0 & 1.4138 & 0 \\
0 & 0 & 1.3535
\end{array}\right]
\end{aligned}
$$

There, it follows from Theorem 1 the DRNN (2) with given parameters is globally exponentially stable, which is further verified by the simulation result given in Fig. 1.

\section{Conclusions}

In this paper, we have considered the analysis problem of exponential stability for a class of discrete-time recurrent neural networks (DRNNs) with time delays. The activation functions have been assumed to be neither differentiable nor strict monotonic. Furthermore, the description of the activation functions has been 


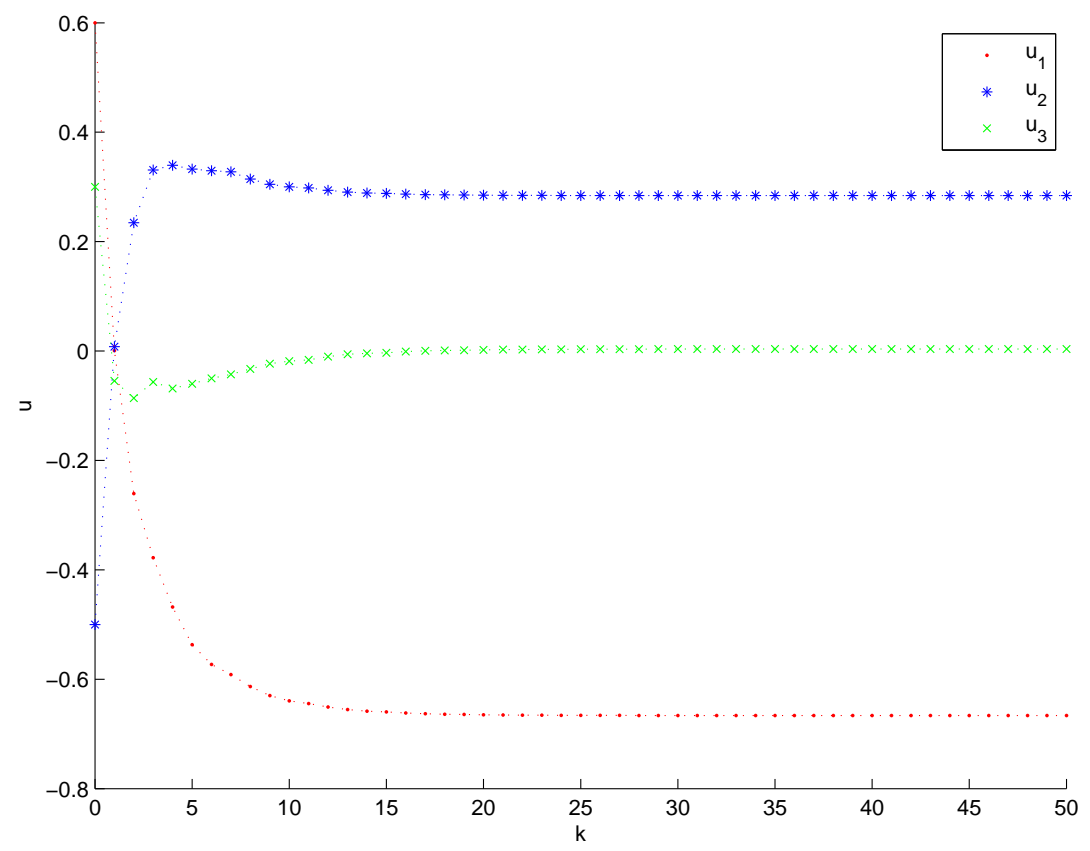

Fig. 1. State trajectory of the DRNN in the example.

more general than the recently commonly used Lipschitz conditions. We have first proved the existence of the equilibrium point, and then by employing an Lyapnuov-Krasovskii functional, a unified linear matrix inequality (LMI) approach has been developed to establish sufficient conditions for the DRNNs to be globally exponentially stable. It has been shown that the delayed DRNNs are globally exponentially stable if a certain LMI is solvable, where the feasibility of such an LMI can be easily checked by using the numerically efficient Matlab LMI Toolbox. A simulation example has been presented to show the usefulness of the derived LMIbased stability condition.

\section{REFERENCES}

[1] Arik, S. (2000) Stability analysis of delayed neural networks. IEEE Transactions on Circuits Systems -I, 47, 1089-1092.

[2] Cao, J. and Song, Q. (2006) Stability in Cohen-Grossberg-type bidirectional associative memory neural networks with timevarying delays. Nonlinearity, 19(7), 1601-1617.

[3] Cao, J. and Ho, D. W. C. (2005) A general framework for global asymptotic stability analysis of delayed neural networks based on LMI approach. Chaos, Solitons 8 Fractals, 24(5), 1317-1329.

[4] Chen, H.-C., Hung, Y.-C., Chen, C.-K., Liao, T.-L. and Chen, C.-K. (2006) Image-processing algorithms realized by discretetime cellular neural networks and their circuit implementations, Chaos, Solitons 86 Fractals, 29(5), 1100-1108.

[5] Chen, W.-H., Lu, X. and Liang D.-Y. (2006) Global exponential stability for discrete-time neural networks with variable delays, Physics Letters A, 358(3), 186-198.

[6] Fridman, E. and Shaked, U. (2005) Stability and guaranteed cost control of uncertain discrete delay systems, International Journal of Control, 78(4), 235-246.

[7] Gao, H., Lam, J., Wang, C. and Wang, Y. (2004) Delay-dependent output-feedback stabilisation of discrete-time systems with time-varying state delay, IEE Proceedings - Control Theory and Applications, 151(6), 691-698.

[8] Gao, H. and Wang, C. (2005) LMI approach to robust filtering for discrete time-delay systems with nonlinear disturbances, Asian Journal of Control, 7(2), 81-90.

[9] Gao, H., Lam, J., Xie, L. and Wang, C. (2005) New approach to mixed $H_{2} / H_{\infty}$ filtering for polytopic discrete-time systems, IEEE Transactions on Signal Processing, 53(8), 3183-3192.

[10] Gicquel, N., Anderson, J. S. and Kevrekidis, I. G. (1998) Noninvertibility and resonance in discrete-time neural networks for time-series processing, Physics Letters A, 238(1), 8-18.

[11] Hu, S. and Wang, J. (2006) Global robust stability of a class of discrete-time interval neural networks, IEEE Trans. Circuits and Systems I - Regular Papers, 53(1), 129-138. 
[12] Liang, J., Cao, J. and Lam, J. (2005) Convergence of discrete-time recurrent neural networks with variable delay, Int. J. Bifurcation and Chaos, 15(2), 581-595.

[13] Liang, J., Cao, J. and Ho, D.W.C. (2005) Discrete-time bidirectional associative memory neural networks with variable delays, Physics Letters A, 335(2-3), 226-234.

[14] Mohamad, S. and Naim, A. (2002) Discrete-time analogues of integrodifferential equations modelling bidirectional neural networks, J. Comput. Appl. Math. 138, 1-20.

[15] Mohamad, S. and Gopalsamy, K. (2003) Exponential stability of continuous-time and discrete-time cellular neural networks with delays, Applied Mathematics and Computation, 135(1), 17-38.

[16] Song, Q. and Cao, J. (2006) Periodic solutions and its exponential stability of reaction-diffusion recurrent neural networks with continuously distributed delays, Nonlinear Analysis - Real World Applications, 7(1), 65-80.

[17] Stuart, A. and Humphries, A. (1996) Dynamical Systems and Numerical Analysis, Cambridge University Press, Cambridge, 1996.

[18] Tan, K., Tang, H. and Yi, Z. (2004) Global exponential stability of discrete-time neural networks for constrained quadratic optimization, Neurocomputing, 56, 399-406.

[19] Wang, L. and $\mathrm{Xu}, \mathrm{Z}$. (2006) Sufficient and necessary conditions for global exponential stability of discrete-time recurrent neural networks, IEEE Trans. Circuits and Systems I-Regular Papers, 53(6), 1373-1380.

[20] Wang, Z., Liu, Y. and Liu, X. (2005) On global asymptotic stability of neural networks with discrete and distributed delays, Physics Letters A, 345(4-6), 299-308.

[21] Wang, Z., Liu, Y., Li, M. and Liu, X. (2006) Stability analysis for stochastic Cohen-Grossberg neural networks with mixed time delays, IEEE Trans. Neural Networks, 17(3), 814-820.

[22] Wang, Z., Liu, Y., Fraser, K. and Liu, X. (2006) Stochastic stability of uncertain Hopfield neural networks with discrete and distributed delays, Physics Letters A, 354(4), 288-297.

[23] Wang, Z., Shu, H., Fang, J. and Liu, X. (2006) Robust stability for stochastic Hopfield neural networks with time delays, Nonlinear Analysis: Real World Applications, 7(5), 1119-1128.

[24] Wang, Z., Liu, Y., Yu, L. and Liu, X. (2006) Exponential stability of delayed recurrent neural networks with Markovian jumping parameters, Physics Letters A, 356(4-5), 346-352.

[25] Xiang, H., Yan, K. and Wang, B. (2005) Existence and global stability of periodic solution for delayed discrete high-order Hopfield-type neural networks, Discrete Dynamics in Nature and Society, 3, 281-297.

[26] Xiong, W. and Cao, J. (2005) Global exponential stability of discrete-time Cohen-Grossberg neural networks, Neurocomputing, 64, 433-446.

[27] Yu, W. (2004) Nonlinear system identification using discrete-time recurrent neural networks with stable learning algorithms, Information Sciences, 158, 131-147.

[28] Yuan, Z., Hu, D. and Huang, L. (2005) Stability and bifurcation analysis on a discrete-time neural network, J. Computational and Applied Mathematics, 177(1), 89-100.

[29] Zhao, H. and Wang, L. (2006) Stability and bifurcation for discrete-time Cohen-Grossberg neural network, Applied Mathematics and Computation, 179(2), 787-798.

[30] Zou, L. and Zhou, Z. (2006) Periodic solutions for nonautonomous discrete-time neural networks, Applied Mathematics Letters, 19(2), 174-185. 